\title{
USO DE DIFERENTES ALTERNATIVAS PARA VIABILIZAR A RELAÇÃO HIPSOMÉTRICA NO POVOAMENTO FLORESTAL ${ }^{1}$
}

Thelma Shirlen Soares² ${ }^{2}$ José Roberto Soares Scolforo ${ }^{3}$, Sebastião Oswaldo Ferreira ${ }^{4}$ e José Márcio de Mello ${ }^{3}$

\begin{abstract}
RESUMO - Este estudo teve como objetivo testar diversos modelos hipsométricos tradicionais e genéricos selecionados na literatura florestal, observando-se seus ajustes e comportamentos em diferentes agrupamentos de variáveis independentes que caracterizam um povoamento florestal. Esses modelos hipsométricos foram ajustados, sendo o critério de seleção da equação mais precisa através do coeficiente de determinação ajustado e erro-padrão residual. Para identificar se equações selecionadas para cada situação são estatisticamente diferentes, adotou-se o delineamento inteiramente casualizado no esquema de parcelas subdivididas. Nos casos em que foi detectado diferença significativa na análise de variância, aplicou-se o teste de médias de Scott e Knott, constando que o ajuste por parcela utilizando modelos tradicionais é o procedimento ideal para estimar a altura das árvores. Porém, o ajuste do modelo genérico propiciou boas estimativas, indicando a possibilidade de seu uso em substituição aos modelos tradicionais.
\end{abstract}

Palavras chave: Modelos de regressão, altura e eucalipto.

\section{USE OF DIFFERENT ALTERNATIVES TO ALLOW THE USE OF THE HYPSOMETRIC RELATION IN FOREST STANDS}

\begin{abstract}
This study aimed to test several traditional and generic hypsometric models, analyzing the adjustments and behavior in different groupings of independent variables that characterize a forest stand. Traditional and generic hypsometric models were determined, the most exact equation was selected through the determination coefficient and residual standard error. To verify whether equations selected for each situation are different, a complete randomized experimental design in split plot arrangement was adopted. The Scott \& Knott mean test was applied for the cases where it was detected significant difference in the variance analysis were detected. It was verified that the adjustment per plot using traditional models is the ideal procedure to estimate tree height. However, the adjustment of the generic model gave good estimates, indicating the possibility of its use in substitution to the traditional models.
\end{abstract}

Key words: Regression models, height, eucalyptus.

\footnotetext{
${ }^{1}$ Recebido em 13.02.2003 e aceito para publicação em 10.08.2004.

${ }^{2}$ Programa de Pós-Graduação em Ciência Florestal da Universidade Federal de Viçosa. Viçosa, MG. E.mail: <thelsoares@ vicosa.ufv.br>.

${ }^{3}$ Departamento de Ciências Florestais da Universidade Federal de Lavras. Lavras, MG.

${ }^{4}$ Ksti Serviços Técnicos Ltda. Lavras, MG.
} 


\section{INTRODUÇÃO}

A modelagem dos fenômenos que retratam o desenvolvimento da floresta tem apresentado crescente desenvolvimento nas últimas décadas. Os modelos biométricos de prognose estão sendo sofisticados, a fim de propiciar informações cada vez mais detalhadas sobre o povoamento florestal.

Em contraposição a esse desenvolvimento, uma série de questões sobre a coleta de dados pode ser objeto de questionamento. Uma delas é sobre a precisão, custo e necessidade de medição da altura das árvores. Assim, busca-se saber qual é o melhor procedimento para que custo e precisão sejam otimizados, bem como analisar se a relação hipsométrica é afetada pela espécie, idade, sítio e espaçamento (CARDOSO et al., 1989).

Avaliando a influência de tais fatores, é possível analisar a viabilidade de uma equação genérica que permita estimativas de altura para um conjunto de dados oriundos de povoamentos com características heterogêneas. Assim, de acordo com Cardoso (1989), será possível eliminar o trabalho de ajuste e seleção de equações mais adequadas para cada parcela ou povoamento, reduzindo-se o custo de processamento dos dados.

Segundo Barros et al. (2004), vários modelos estatísticos podem adequar-se à relação hipsométrica. Porém, o uso indiscriminado de tal relação pode levar a erros consideráveis, uma vez que vários fatores influenciam tal relação, como posição sociológica, sítio, idade, densidade e práticas silviculturais em geral.

Diante da avaliação da influência de tais fatores, deve-se, assim, estudar a viabilidade da aplicação de equações genéricas que permitam estimativas para um conjunto de dados originados de povoamentos florestais com características silviculturais diferentes. Dessa forma, os custos de processamento dos dados diminuirão, eliminando o trabalho de ajuste e seleção das equações mais adequadas para cada unidade amostral.

Nesse contexto, este estudo objetivou avaliar a acuracidade de modelos hipsométricos tradicionais e genéricos e obter informações mais definidas para a aplicação correta das relações hipsométricas em florestas plantadas.

R. Árvore, Viçosa-MG, v.28, n.6, p.845-854, 2004

\section{MATERIAL E MÉTODOS}

\subsection{Fonte de dados}

Este estudo foi desenvolvido com dados de um povoamento de Eucalyptus grandis W. Hill ex Maiden pertencente à empresa Votorantim Celulose e Papel S/A, localizado no município de Guatapará, Estado de São Paulo.

A área amostral foi composta de 22 parcelas, distribuídas em três diferentes classes de sítio e com idade variando de 4 a 7,1 anos (Quadro 1).

Visando obter a altura real das árvores do povoamento, foram abatidas todas as árvores das parcelas, perfazendo um total de 20 árvores por parcela, das quais foram medidos os diâmetros a 1,30 m do solo (dap) e a altura total $(h)$.

As informações dendrométricas obtidas foram agrupadas em diferentes categorias: idade, índice de sítio e idade por índice de sítio, além do agrupamento de todas as parcelas, criando-se arquivos que contivessem pares de valores dap- $h$.

\subsection{Modelos hipsométricos}

Para cada agrupamento de dados foram ajustados, utilizando-se o programa estatístico SAS (SAS, 1996), modelos hiposmétricos tradicionais e genéricos.

O critério de seleção da equação mais precisa foi o conjunto das seguintes medidas de precisão: maior coeficiente de determinação ajustado ( $\mathrm{R}^{2}$ aj. $)$ e menor erro-padrão residual $\left(\mathrm{S}_{\mathrm{yx}}\right)$.

\subsubsection{Modelos tradicionais}

São considerados tradicionais os modelos hipsométricos que descrevem as alturas das árvores em função apenas dos diâmetros medidos a 1,30 m do solo (dap). Os modelos ajustados, os quais foram apresentados por Scolforo (1993), são descritos no Quadro 2.

\subsubsection{Modelos hipsométricos genéricos}

Em populações florestais onde se conhecem o índice de sítio, suas estruturas diamétrica e de altura, a densidade e a altura, podem-se obter estimativas da altura média através dos modelos hipsométricos, os quais são denominados genéricos (SCOLFORO, 1993).

Neste estudo foram testados 14 modelos genéricos (Quadro 3), os quais foram descritos por Scolforo (1993), Cardoso (1989), Abreu (2000) e Barros et al. (2004), dentre outros. 
Quadro 1 - Características gerais do povoamento em estudo Table 1 - General characteristics of the forest stand

\begin{tabular}{|c|c|c|c|c|c|c|c|c|}
\hline Parcela & NARV & $\begin{array}{c}\mathrm{S} \\
(\mathrm{m})\end{array}$ & $\begin{array}{l}\mathrm{D}_{\max } \\
(\mathrm{cm})\end{array}$ & $\begin{array}{l}\mathrm{D}_{\min } \\
(\mathrm{cm})\end{array}$ & $\begin{array}{l}\mathrm{Hd} \\
(\mathrm{m})\end{array}$ & $\begin{array}{c}\mathrm{I} \\
(\operatorname{anos})\end{array}$ & $\begin{array}{c}\mathrm{G} \\
\left(\mathrm{m}^{2} / \mathrm{ha}\right)\end{array}$ & $\begin{array}{c}\mathrm{D}_{\mathrm{g}} \\
(\mathrm{cm})\end{array}$ \\
\hline 1 & 1140,5 & 28,5 & 17,5 & 7,0 & 24,0 & 4,7 & 17,1 & 12,3 \\
\hline 2 & 1075,3 & 28,5 & 21,5 & 4,9 & 25,9 & 4,6 & 16,0 & 13,8 \\
\hline 3 & 1133,8 & 25,5 & 16,9 & 6,5 & 20,4 & 4,6 & 12,2 & 11,7 \\
\hline 4 & 981,8 & 25,5 & 16,5 & 6,5 & 20,1 & 4,4 & 11,6 & 12,2 \\
\hline 5 & 1141,6 & 22,5 & 19,5 & 7,2 & 23,2 & 6,8 & 18,4 & 14,3 \\
\hline 6 & 913,2 & 25,5 & 20,0 & 7,5 & 24,3 & 7,1 & 15,6 & 14,7 \\
\hline 7 & 1187,4 & 25,5 & 22,5 & 4,7 & 26,9 & 7,0 & 15,2 & 12,8 \\
\hline 8 & 1785,2 & 28,5 & 19,0 & 5,5 & 26,4 & 6,0 & 21,8 & 12,5 \\
\hline 9 & 1262,6 & 25,5 & 19,5 & 9,8 & 25,5 & 6,8 & 20,4 & 14,3 \\
\hline 10 & 1646,1 & 28,5 & 18,9 & 7,5 & 27,5 & 6,4 & 19,2 & 12,2 \\
\hline 11 & 1831,5 & 25,5 & 19,0 & 5,9 & 24,6 & 6,1 & 20,0 & 11,8 \\
\hline 12 & 1379,7 & 28,5 & 21,0 & 4,8 & 26,4 & 6,2 & 20,4 & 13,7 \\
\hline 13 & 1034,2 & 28,5 & 20,5 & 4,9 & 23,5 & 4,2 & 17,5 & 14,7 \\
\hline 14 & 1145,5 & 25,5 & 17,9 & 6,8 & 22,1 & 4,4 & 16,0 & 13,3 \\
\hline 15 & 1070,2 & 25,5 & 16,3 & 6,5 & 19,8 & 4,1 & 12,5 & 12,2 \\
\hline 16 & 1111,1 & 25,5 & 16,5 & 7,9 & 20,0 & 4,0 & 16,4 & 13,7 \\
\hline 17 & 1272,3 & 25,5 & 21,0 & 5,5 & 23,6 & 6,3 & 16,0 & 12,7 \\
\hline 18 & 1732,4 & 25,5 & 19,0 & 5,8 & 23,1 & 6,2 & 20,7 & 12,3 \\
\hline 19 & 1501,5 & 28,5 & 22,5 & 6,9 & 26,1 & 4,9 & 17,8 & 12,3 \\
\hline 20 & 1564,9 & 25,5 & 14,5 & 5,5 & 19,8 & 4,8 & 14,4 & 10,8 \\
\hline 21 & 1274,2 & 25,5 & 17,8 & 6,2 & 21,7 & 4,6 & 14,3 & 12,0 \\
\hline 22 & 1099,7 & 25,5 & 17,2 & 4,9 & 20,4 & 4,7 & 10,6 & 11,1 \\
\hline
\end{tabular}

Em que: $\mathrm{NARV}=$ número de árvores por hectare, $\mathrm{S}=$ índice de sítio, $\mathrm{D}_{\max }=$ maior diâmetro da parcela, $\mathrm{D}_{\min }=$ menor diâmetro da parcela, $\mathrm{Hd}$ = altura média das árvores dominantes, $\mathrm{I}=$ idade, $\mathrm{G}=$ área basal e $\mathrm{D}_{\mathrm{g}}=$ diâmetro médio quadrático.

Quadro 2 - Modelos tradicionais de relação hipsométrica Table 2 - Traditional models of hypsometric relationship

\begin{tabular}{ccc}
\hline Modelo & Autor & Forma de Ajuste \\
\hline 1 & Modelo Parabólico & $h=\beta_{0}+\beta_{1}$ dap $+\beta_{2}$ dap $^{2}+\varepsilon_{\mathrm{i}}$ \\
2 & Stoffels & $\ln (h)=\beta_{0}+\beta_{1} \ln \left(\right.$ dap $+\varepsilon_{\mathrm{i}}$ \\
3 & Curtis & $\ln (h)=\beta_{0}+\beta_{1}$ dap $^{-1}+\varepsilon_{\mathrm{i}}$ \\
4 & Prodan & $h=\left(\right.$ dap $^{2} / \beta_{0}+\beta_{1} d a p+\beta_{2}$ dap $\left.^{2}\right)+\varepsilon_{\mathrm{i}}$ \\
5 & Modelo da Linha Reta & $h=\beta_{0}+\beta_{1}$ dap $+\varepsilon_{\mathrm{i}}$ \\
6 & Petterson & $1 / \sqrt[3]{h-1,3}=\beta_{0}+\beta_{1}$ dap $^{-1}+\varepsilon_{\mathrm{i}}$ \\
\hline
\end{tabular}

Em que: $h=$ altura total da árvore $(\mathrm{m}) ;$ dap $=$ diâmetro medido a $1,30 \mathrm{~m}$ do solo $(\mathrm{cm}) ; \ln =\log$ aritmo neperiano; $\beta_{o}, \beta_{l}$ $e \beta_{2}=$ parâmetros a serem estimados; e $\varepsilon_{i}=$ erro da estimativa 
Quadro 3 - Modelos genéricos de relação hipsométrica

Table 3 - Generic models of hypsometric relationship

\begin{tabular}{|c|c|c|}
\hline Modelo & Autor & Forma de Ajuste \\
\hline 1 & Scolforo & $\ln (h)=\beta_{0}+\beta_{1} \ln (h \mathrm{~d})+\beta_{2} \ln \left(\mathrm{D}_{\mathrm{g}} /\right.$ dap $)+\beta_{3}[1 /($ I/dap $)]+\beta_{4} \ln \left(\right.$ dap $\left.^{-1}\right)+\varepsilon_{\mathrm{i}}$ \\
\hline 2 & Scolforo & $\ln (h)=\beta_{0}+\beta_{1} I^{-1}+\beta_{2} \ln (h d)+\beta_{3} \ln (G)+\beta_{4}(I x d a p)^{-1}+\varepsilon_{\mathrm{i}}$ \\
\hline 3 & Scolforo & $\ln (h)=\beta_{0}+\beta_{1} \ln (h d)+\beta_{2} I^{-1}+\beta_{3} \ln \left(D_{g} /\right.$ dap $)+\beta_{4} \ln \left(D_{g} \mathrm{x} I\right)+\varepsilon_{\mathrm{i}}$ \\
\hline 4 & Scolforo & $\ln (h)=\beta_{0}+\beta_{1} \ln (h d)+\beta_{2}\left(d a p^{-1}-D_{\max }^{-1}\right)+\beta_{3} \ln (N /$ dap $)+\beta_{4}(\text { Ixdap })^{-1}+\varepsilon_{\mathrm{i}}$ \\
\hline 5 & Scolforo & $\ln (h)=\beta_{0}+\beta_{1} \ln (h d)+\beta_{2} d a p^{-1}+\beta_{3} \ln (N /$ dap $)+\beta_{4}(\text { Ixdap })^{-1}+\varepsilon_{\mathrm{i}}$ \\
\hline 6 & Amateis et al. & $\ln (h)=\beta_{0}+\beta_{1} I^{-1}+\beta_{2} \ln (h d)+\beta_{3} \ln (G)+\beta_{4} \ln (N)+\beta_{5} d a p^{-1}+\varepsilon_{\mathrm{i}}$ \\
\hline 7 & Cao et al. & $\ln (h)=\beta_{0}+\beta_{1} I^{-1}+\beta_{2} \ln (h d)+\beta_{3} \ln (G)+\varepsilon_{\mathrm{i}}$ \\
\hline 8 & Clutter e Bennett & $\ln (h)=\beta_{0}+\beta_{1} \mathrm{~S}+\beta_{2} N+\beta_{3} I^{-1}+\beta_{4} d a p^{-1}+\varepsilon_{\mathrm{i}}$ \\
\hline 9 & Lenhart & $\ln (h)=\beta_{0}+\beta_{1} \ln (h d)+\beta_{2} I^{-1}+\beta_{3} \ln (N \mathrm{x}$ dap $)+\beta_{4}(\operatorname{Ix} \text { dap })^{-1}+\varepsilon_{\mathrm{i}}$ \\
\hline 10 & Lenhart & $\ln (h)=\ln (h d)+\beta_{0}+\left[\ln (\right.$ dap $\left.)-\ln \left(D_{\max }\right)\right] \times\left[\beta_{1}+\beta_{2} \ln \left(D_{g}\right)\right]+\varepsilon_{\mathrm{i}}$ \\
\hline 11 & Burkhart & $\ln (h)=\ln (h d) /\left\{\beta_{0}+\left[\right.\right.$ dap $\left.\left.^{-1}-D_{\max }^{-1}\right] \times\left[\beta_{1}+\beta_{2} \ln (G)+\beta_{3} I^{-1}+\beta_{4} S\right]\right\}+\varepsilon_{\mathrm{i}}$ \\
\hline 12 & Amateis et al. & $\ln (h)=\ln (h d) /\left\{\beta_{0}+\left[\right.\right.$ dap $\left.\left.^{-1}-D_{\max }^{-1}\right] \times\left[\beta_{1}+\beta_{2} \ln (N)+\beta_{3} I^{-1}+\beta_{4} S\right]\right\}+\varepsilon_{\mathrm{i}}$ \\
\hline 13 & Lenhart e Clutter & $\ln (h)=\beta_{0}+\beta_{1}\left[\right.$ dap $\left.^{-1}-D_{\max }^{-1}\right]+\beta_{2}\left[I^{-1} \times\left(\right.\right.$ dap $\left.\left.^{-1}-D_{\max }^{-1}\right)\right]+\beta_{3}\left\{\left[\right.\right.$ dap $\left.\left.^{-1}-D_{\max }{ }^{-1}\right] \times \ln (N)\right\}+\varepsilon_{\mathrm{i}}$ \\
\hline 14 & Lenhart & $\ln (h)=\ln (h d)+\beta_{0}+\left[\ln (\right.$ dap $\left.)-\ln \left(D_{\max }\right)\right] \times\left[\beta_{1}+\beta_{2} \ln (I)+\beta_{3} \ln (h d / I)+\beta_{4} \ln (N)\right]+\varepsilon_{\mathrm{i}}$ \\
\hline
\end{tabular}

Em que: $h=$ altura total da árvore $(\mathrm{m}) ;$ dap = diâmetro medido a $1,30 \mathrm{~m}$ do solo $(\mathrm{cm}) ; h d=$ altura média das árvores dominantes $(\mathrm{m})$; $D_{g}=$ diâmetro médio quadrático $(\mathrm{cm}) ; I=$ idade $(\operatorname{anos}) ; D_{m a x}=$ maior diâmetro do povoamento $(\mathrm{cm}) ; G=$ área basal $\left(\mathrm{m}^{2} / \mathrm{ha}\right) ; S=$ índice de sítio $(\mathrm{m}) ; N=$ número de árvores por hectare; $l n=\log$ aritmo neperiano; $\beta_{0}, \beta_{1}, \beta_{2}, \beta_{3}, \beta_{4} e \beta_{5}=$ parâmetros a serem estimados; e $\varepsilon_{i}=$ erro da estimativa.

\subsection{Eficiência dos modelos ajustados}

Após ajustar e selecionar os modelos mais eficientes, aplicou-se um teste para verificar se as equações selecionadas para as diferentes situações estudadas diferiam estatisticamente entre si. Para tal, utilizouse um delineamento inteiramente casualizado, no esquema de parcelas subdivididas. O modelo estatístico empregado no delineamento é dado por:

$$
\mathrm{Y}_{\mathrm{ijk}}=\mu+\mathrm{T}_{\mathrm{i}}+\delta_{\mathrm{ij}}+\mathrm{T}_{\mathrm{k}}{ }_{\mathrm{k}}+\mathrm{TT}^{\prime}{ }_{\mathrm{ik}}+\varepsilon_{\mathrm{ijk}}
$$

em que:

$\mathrm{Y}_{\mathrm{ijk}}=$ valor observado na ik-ésima subparcela, na
j-ésima repetição;

$\mu=$ média geral do experimento;

$\mathrm{T}_{\mathrm{i}}=$ efeito do i-ésimo nível do tratamento $\mathrm{T}(\mathrm{i}=1$, $2, \ldots, 22)$

$\delta_{\mathrm{ij}}=$ efeito residual das parcelas;

$\mathrm{T}^{\prime}{ }_{\mathrm{k}}=$ efeito do k-ésimo nível do tratamento T' $(\mathrm{k}=$ $1,2, \ldots, 7)$
$\mathrm{TT}{ }_{\mathrm{ik}}=$ efeito da interação do i-ésimo nível do tratamento $\mathrm{T}$ com o k-ésimo nível do tratamento T'; e

$\varepsilon_{\mathrm{ijk}}=$ efeito residual das subparcelas.

Neste estudo, cada parcela compôs um experimento, as situações nas quais foram testados os modelos compuseram os tratamentos e as árvores, as repetições. Foram considerados sete tratamentos: $\mathrm{T}_{1}=$ testemunha (altura real); $\mathrm{T}_{2}=$ altura obtida para cada parcela pelo modelo tradicional; $\mathrm{T}_{3}=$ altura obtida para cada idade pelo modelo tradicional; $\mathrm{T}_{4}=$ altura obtida para cada sítio pelo modelo tradicional; $\mathrm{T}_{5}=$ altura obtida para cada agrupamento de sítio e idade pelo modelo tradicional; $\mathrm{T}_{6}=$ altura obtida para o agrupamento de todas as parcelas pelo modelo tradicional; $\mathrm{e}_{7}=$ altura obtida pelo modelo genérico.

Buscou-se, através desse teste, identificar nas diferentes situações se havia diferença entre os modelos para a estimativa das alturas em cada sortimento considerado. No caso em que a interação foi significativa a $95 \%$ de probabilidade de acerto, procedeu-se ao desdobramento, aplicando o teste de Scott e Knott (1974). 
Tanto a análise de variância quanto o teste de médias foram realizados com o software SISVAR, desenvolvido por Ferreira (1999).

\section{RESULTADOS E DISCUSSÃO}

\subsection{Seleção das equações tradicionais}

As situações para as quais se ajustaram os modelos tradicionais foram: para cada parcela, para cada sítio, para cada idade, para cada agrupamento de sítio e idade e para o agrupamento de todas as parcelas.

\section{a) Ajustes por parcela}

No Quadro 4 são apresentados os resultados pertinentes aos ajustes, com os respectivos parâmetros estatísticos de precisão. Pode-se observar, nesse quadro, que as equações de Curtis (parcelas 1, 4, 6, 7, 12, 15, 16 e 20), Prodan (parcelas 3, 8 10, 11, 13 e 18), Parabólica (parcelas 2, 5, 9, 14, 17, 19 e 22) e de Stoffels (parcela 21) foram as que apresentaram melhor desempenho nas parcelas ajustadas.

\section{b) Ajustes por idade}

As equações selecionadas para cada idade são apresentadas no Quadro 5, onde é possível observar que as equações que apresentaram melhores ajustes foram as de Curtis (para as idades de 4,$0 ; 4,1 ; 4,4 ; 4,8$; 7,$0 ;$ e 7,1 anos), Prodan (nas idades de 4,2;6,0;6,1; 6,4; e 6,8 anos), Parabólica (nas idades de 4,9; 6,5 e 6,3 anos) e de Stoffels (para as idades de 4,6 e 4,7 anos).

Quadro 4 - Parâmetros estimados e medidas de precisão das equações hipsométricas tradicionais selecionadas para as parcelas de Eucalyptus grandis

Table 4 - Estimated parameters and precision measures for the traditional hypsometric equations selected for Eucalyptus grandis plots

\begin{tabular}{|c|c|c|c|c|}
\hline \multirow[t]{2}{*}{ Parcela } & \multirow[t]{2}{*}{ Equação selecionada } & \multirow{2}{*}{$\begin{array}{c}\mathrm{R}^{2} \text { aj. } \\
(\%)\end{array}$} & \multicolumn{2}{|c|}{$\mathrm{S}_{\mathrm{yx}}$} \\
\hline & & & $(\mathrm{m})$ & $(\%)$ \\
\hline 1 & $\ln (\hat{h})=3,59019-6,99694 d a p^{-1}$ & 79,82 & $\pm 1,49$ & $\pm 7,5$ \\
\hline 2 & $\hat{h}=-2,24435+2,75424$ dap $-0,06520$ dap $^{2}$ & 90,99 & $\pm 1,42$ & $\pm 6,5$ \\
\hline 3 & $\hat{h}=\left(d_{a p} / 3,38044-0,36655 d a p+0,06068 d a p^{2}\right)$ & 99,84 & $\pm 0,75$ & $\pm 4,4$ \\
\hline 4 & $\ln (\hat{h})=3,43592-6,60369 d a p^{-1}$ & 75,41 & $\pm 1,40$ & $\pm 8,0$ \\
\hline 5 & $\hat{h}=4,50395+1,86125$ dap $-0,04606 d_{a p}^{2}$ & 71,17 & $\pm 1,19$ & $\pm 5,6$ \\
\hline 6 & $\ln (\hat{h})=3,55446-6,92956 d a p^{-1}$ & 89,59 & $\pm 1,08$ & $\pm 5,2$ \\
\hline 7 & $\ln (\hat{h})=3,63310-6,55650$ dap $^{-1}$ & 88,92 & $\pm 1,57$ & $\pm 7,1$ \\
\hline 8 & $\hat{h}=\left(d a p^{2} / 1,77590-0,07494 d a p+0,03622 d a p^{2}\right)$ & 99,73 & $\pm 1,32$ & $\pm 5,7$ \\
\hline 9 & $\hat{h}=-8,96023+4,07650 d a p+0,12288 d a p^{2}$ & 92,51 & $\pm 0,53$ & $\pm 2,3$ \\
\hline 10 & $\hat{h}=\left(d a p^{2} / 1,57132-0,01414 d a p+0,03384 d a p^{2}\right)$ & 99,82 & $\pm 1,01$ & $\pm 4,6$ \\
\hline 11 & $\hat{h}=\left(d a p^{2} / 1,53294+0,01813 d a p+0,03504 d a p^{2}\right)$ & 99,85 & $\pm 0,86$ & $\pm 4,2$ \\
\hline 12 & $\ln (\hat{h})=3,61537-6,34396 d a p^{-1}$ & 96,32 & $\pm 0,92$ & $\pm 4,2$ \\
\hline 13 & $\hat{h}=\left(d a p^{2} / 0,33210+0,34302 d a p+0,02365 d a p^{2}\right)$ & 99,71 & $\pm 1,20$ & $\pm 5,9$ \\
\hline 14 & $\hat{h}=5,28794+1,79985-0,04807 d a p^{2}$ & 78,26 & $\pm 0,86$ & $\pm 4,2$ \\
\hline 15 & $\ln (\hat{h})=3,30753-5,09548 d a p^{-1}$ & 83,81 & $\pm 0,92$ & $\pm 5,3$ \\
\hline 16 & $\ln (\hat{h})=3,36521-5,91162 d a p^{-1}$ & 85,97 & $\pm 0,66$ & $\pm 3,5$ \\
\hline 17 & $\hat{h}=0,01633+2,3777$ dap $-0,05938 d^{2} a p^{2}$ & 82,77 & $\pm 1,42$ & $\pm 7,7$ \\
\hline 18 & $\hat{h}=\left(d a p^{2} / 0,31621+0,26997 d a p+0,02745 d a p^{2}\right)$ & 99,55 & $\pm 1,34$ & $\pm 7,2$ \\
\hline 19 & $\hat{h}=1,68722+2,2170$ dap $-0,04946$ dap $^{2}$ & 96,63 & $\pm 0,70$ & $\pm 3,4$ \\
\hline 20 & $\ln (\hat{h})=3,24383-3,93835$ dap $^{-1}$ & 89,87 & $\pm 0,64$ & $\pm 3,8$ \\
\hline 21 & $\ln (\hat{h})=1,68228+0,49977 \ln ($ dap $)$ & 88,37 & $\pm 0,83$ & $\pm 4,7$ \\
\hline 22 & $\hat{h}=4,26524+1,68779$ dap $-0,04082$ dap $^{2}$ & 91,51 & $\pm 0,83$ & $\pm 4,7$ \\
\hline
\end{tabular}

R. Árvore, Viçosa-MG, v.28, n.6, p.845-854, 2004 
Quadro 5 - Parâmetros estimados e medidas de precisão das equações hipsométricas tradicionais selecionadas, para Eucalyptus grandis, por idade

Table 5 - Estimated parameters and precision measures for the traditional hypsometric equations selected for Eucalyptus grandis perage

\begin{tabular}{|c|c|c|c|c|}
\hline \multirow[t]{2}{*}{ Idade } & \multirow[t]{2}{*}{ Equação Selecionada } & \multirow{2}{*}{$\begin{array}{c}\mathrm{R}^{2} \mathrm{aj} . \\
(\%)\end{array}$} & \multicolumn{2}{|c|}{$\mathrm{S}_{\mathrm{yx}}$} \\
\hline & & & $(\mathrm{m})$ & $(\%)$ \\
\hline 4,0 & $\ln (\hat{h})=3,36521-5,91162$ dap $^{-1}$ & 85,97 & $\pm 0,66$ & $\pm 3,5$ \\
\hline 4,1 & $\ln (\hat{h})=3,30753-5,09548 d a p^{-1}$ & 83,81 & $\pm 0,92$ & $\pm 5,3$ \\
\hline 4,2 & $\hat{h}=\left(\right.$ dap $^{2} / 0,33210+0,34302 d a p+0,02365$ dap $\left.^{2}\right)$ & 99,71 & $\pm 1,20$ & $\pm 5,9$ \\
\hline 4,4 & $\ln (\hat{h})=3,45328-6,23616 d a p^{-1}$ & 70,97 & $\pm 1,50$ & $\pm 7,9$ \\
\hline 4,6 & $\ln (\hat{h})=1,30445+0,66728 \ln ($ dap $)$ & 83,30 & $\pm 1,64$ & $\pm 8,7$ \\
\hline 4,7 & $\ln (\hat{h})=1,54863+0,54455 \ln ($ dap $)$ & 83,51 & $\pm 1,43$ & $\pm 7,7$ \\
\hline 4,8 & $\ln (\hat{h})=3,24383-3,93835 d a p^{-1}$ & 89,87 & $\pm 0,64$ & $\pm 3,8$ \\
\hline 4,9 & $\hat{h}=1,68722+2,21700$ dap $-0,04946 d_{a p}^{2}$ & 96,63 & $\pm 0,70$ & $\pm 3,4$ \\
\hline 6,0 & $\hat{h}=\left(\right.$ dap $^{2} / 1,7759-0,07494 d a p+0,03622$ dap $\left.^{2}\right)$ & 99,73 & $\pm 1,32$ & $\pm 5,7$ \\
\hline 6,1 & $\hat{h}=\left(\right.$ dap $^{2} / 1,53294+0,01813 d a p+0,03504$ dap $\left.^{2}\right)$ & 99,85 & $\pm 0,86$ & $\pm 4,2$ \\
\hline 6,2 & $\hat{h}=0,48298+2,30389$ dap $-0,05258 d a p^{2}$ & 83,77 & $\pm 1,74$ & $\pm 8,5$ \\
\hline 6,3 & $\hat{h}=0,01633+2,3777 d a p-0,05938 d a p^{2}$ & 82,77 & $\pm 1,42$ & $\pm 7,7$ \\
\hline 6,4 & $\hat{h}=\left(d a p^{2} / 1,57132-0,01414 d a p+0,03384 d a p^{2}\right)$ & 99,82 & $\pm 1,01$ & $\pm 4,6$ \\
\hline 6,8 & $\hat{h}=\left(d_{a p} / 2,68551-0,19918 d a p+0,04448 d a p^{2}\right)$ & 99,63 & $\pm 1,42$ & $\pm 6,4$ \\
\hline 7,0 & $1(\hat{h})=3,63310-6,55650 \mathrm{dap}^{-1}$ & 88,92 & $\pm 1,57$ & $\pm 7,1$ \\
\hline 7,1 & $\ln (\hat{h})=3,55446-6,92956 d a p^{-1}$ & 89,59 & $\pm 1,08$ & $\pm 5,2$ \\
\hline
\end{tabular}

\section{c) Ajustes por sítio}

O povoamento em estudo apresenta três classes de sítio, sendo para cada uma ajustada uma equação.

No Quadro 6, observa-se que a equação de Curtis foi selecionada para os sítios 25,5 e 28,5, enquanto a equação de Stoffels foi a selecionada para o sítio 22,5.

\section{d) Ajustes por sítio e idade}

No agrupamento dos dados por sítio e idade foram obtidas 20 combinações, para as quais se ajustaram as equações hipsométricas (Quadro 7).

Nos ajustes por sítio e idade, as equações mais eficientes foram as de Curtis, Prodan, Stoffels e Parabólica.

Quadro 6 - Parâmetros estimados e medidas de precisão das equações hipsométricas tradicionais selecionadas, para Eucalyptus grandis, em cada classe de sítio

Table 6 - Estimated parameters and precision measures for traditional hypsometric equations selected for Eucalyptus grandis, per site index

\begin{tabular}{lllll}
\hline Sítio & \multicolumn{1}{c}{ Equação Selecionada } & $\mathrm{R}^{2}$ aj. & \multicolumn{2}{c}{$\mathrm{S}_{\mathrm{yx}}$} \\
\cline { 3 - 5 } & & $(\%)$ & $(\mathrm{m})$ & $(\%)$ \\
\hline 22,5 & $\hat{h}=4,50395+1,86125$ dap $-0,04606$ dap $^{2}$ & 71,17 & $\pm 1,19$ & $\pm 5,6$ \\
25,5 & $\hat{h}=\left(\right.$ dap $^{2} / 0,02400+0,31966$ dap $+0,02481$ dap $\left.^{2}\right)$ & 99,17 & $\pm 1,77$ & $\pm 9,3$ \\
28,5 & $\hat{h}=\left(\right.$ dap $^{2} / 1,67193-0,00722$ dap $+0,03517$ dap $\left.^{2}\right)$ & 99,31 & $\pm 1,82$ & $\pm 8,5$ \\
\hline
\end{tabular}

R. Árvore, Viçosa-MG, v.28, n.6, p.845-854, 2004 
Quadro 7 - Parâmetros estimados e medidas de precisão das equações hipsométricas tradicionais selecionadas para cada combinação de sítio e idade, do povoamento de Eucalyptus grandis

Table 7 - Estimated parameters and precision measures for the traditional hypsometric equations, selected for each combination of index site and age, of Eucalyptus grandis

\begin{tabular}{|c|c|c|c|c|c|}
\hline \multirow[t]{2}{*}{ Sítio } & \multirow[t]{2}{*}{ Idade } & \multirow[t]{2}{*}{ Equação Selecionada } & \multirow{2}{*}{$\begin{array}{c}\mathrm{R}^{2} \text { aj. } \\
(\%)\end{array}$} & \multicolumn{2}{|c|}{$\mathrm{S}_{\mathrm{yx}}$} \\
\hline & & & & $(\mathrm{m})$ & $(\%)$ \\
\hline \multirow[t]{6}{*}{22,5} & 6,8 & $\hat{h}=4,50395+1,86125$ dap $-0,04606$ dap $^{2}$ & 71,17 & $\pm 1,19$ & $\pm 5,6$ \\
\hline & 4,0 & $\ln (\hat{h})=3,36521-5,91162$ dap $^{-1}$ & 85,97 & $\pm 0,66$ & $\pm 3,5$ \\
\hline & 4,1 & $\ln (\hat{h})=3,30753-5,09548 d a p^{-1}$ & 83,81 & $\pm 0,92$ & $\pm 5,3$ \\
\hline & 4,4 & $\ln (\hat{h})=3,45328-6,23616 d a p^{-1}$ & 70,97 & $\pm 1,50$ & $\pm 7,9$ \\
\hline & 4,6 & $\hat{h}=\left(d_{a p}{ }^{2} / 3,38044-0,36655 d a p+0,06068 d a p^{2}\right)$ & 99,84 & $\pm 0,75$ & $\pm 4,4$ \\
\hline & 4,7 & $\hat{h}=4,26524+1,68779$ dap $-0,04082 d_{a p}^{2}$ & 91,51 & $\pm 0,83$ & $\pm 4,7$ \\
\hline \multirow[t]{7}{*}{25,5} & 4,8 & $\ln (\hat{h})=3,24383-3,93835$ dap $^{-1}$ & 89,87 & $\pm 0,64$ & $\pm 3,8$ \\
\hline & 6,1 & $\hat{h}=\left(d a p^{2} / 1,53294+0,01813 d a p+0,03504 d a p^{2}\right)$ & 99,85 & $\pm 0,86$ & $\pm 4,2$ \\
\hline & 6,2 & $\hat{h}=\left(d a p^{2} / 0,31621+0,26997 d a p+0,02745 d a p^{2}\right)$ & 99,55 & $\pm 1,34$ & $\pm 7,2$ \\
\hline & 6,3 & $\hat{h}=0,01633+2,37770 d a p-0,05938 d a p^{2}$ & 82,77 & $\pm 1,42$ & $\pm 7,7$ \\
\hline & 6,8 & $\hat{h}=-8,96023+4,07650 d a p+0,12288 d a p^{2}$ & 99,96 & $\pm 0,53$ & $\pm 2,3$ \\
\hline & 7,0 & $\ln (\hat{h})=3,63310-6,5565 d a p^{-1}$ & 88,92 & $\pm 1,57$ & $\pm 7,1$ \\
\hline & 7,1 & $\ln (\hat{h})=3,55446-6,92956 d a p^{-1}$ & 89,59 & $\pm 1,08$ & $\pm 5,2$ \\
\hline \multirow[t]{7}{*}{28,5} & 4,2 & $\hat{h}=\left(d_{a p^{2}} / 0,33210+0,34302 d a p+0,02365 d_{a p^{2}}\right)$ & 99,71 & $\pm 1,20$ & $\pm 5,9$ \\
\hline & 4,6 & $\ln (\hat{h})=1,30951+0,67375 \ln ($ dap $)$ & 84,34 & $\pm 1,68$ & $\pm 8,5$ \\
\hline & 4,7 & $\ln (\hat{h})=3,59019-6,99694 d a p^{-1}$ & 79,82 & $\pm 1,49$ & $\pm 7,5$ \\
\hline & 4,9 & $\hat{h}=1,68722+2,21700$ dap $-0,04946 d a p^{2}$ & 99,73 & $\pm 1,32$ & $\pm 5,7$ \\
\hline & 6.0 & $\hat{h}=\left(d a p^{2} / 1,7759-0,07494 d a p+0,03622 d a p^{2}\right)$ & 96,63 & $\pm 0,70$ & $\pm 3,4$ \\
\hline & 6,2 & $\ln (\hat{h})=3,61537-6,34396 d a p^{-1}$ & 96,32 & $\pm 0,92$ & $\pm 4,2$ \\
\hline & 6,4 & $\hat{h}=\left(d a p^{2} / 1,57132-0,01414 d a p+0,03384 d a p^{2}\right)$ & 99,82 & $\pm 1,01$ & $\pm 4,6$ \\
\hline
\end{tabular}

\section{e) Ajustes para o agrupamento de todas as parcelas}

Os mesmos seis modelos testados independentemente foram também ajustados para os dados de todas as parcelas agrupados em um único conjunto.

Dos ajustes realizados, a equação de Stoffels foi a que apresentou melhor ajuste:

$$
\begin{aligned}
& \ln (\hat{h})=1,56207+0,56904 \ln (\text { dap }) \\
& \text { com R } \mathrm{R}^{2} \mathrm{aj} .=72,98 \%, \mathrm{~S}_{\mathrm{yx}}= \pm 2,0 \mathrm{~m} \mathrm{e} \pm 10,1 \% .
\end{aligned}
$$

\subsection{Seleção das equações genéricas}

Visando minimizar os erros encontrados nas equações hipsométricas tradicionais, optou-se também pelo ajuste dos modelos genéricos. Estes se diferem dos ajustados anteriormente pela inclusão de algumas variáveis independentes características do povoamento, como altura dominante, idade e diâmetro médio quadrático. Assim, o ajuste foi realizado a partir da união de todas as parcelas numa única base de dados, visando obter variação das variáveis genéricas.

A equação genérica selecionada foi a de Amateis et al.:

$$
\begin{gathered}
\ln (\hat{h})=\frac{\ln (h d)}{0,99389+\left[d a p^{-1}-D_{\max }^{-1}\right] \times[-1,15088+0,21252 \ln (N)} \\
\left.+0,03128 I^{-1}+0,09653 S\right]
\end{gathered}
$$

com $R^{2} a j .=99,97 \%, S_{y x}= \pm 0,87 m$ e $\pm 4,4 \%$.

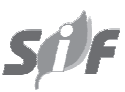

R. Árvore, Viçosa-MG, v.28, n.6, p.845-854, 2004 


\subsection{Eficiência das equações selecionadas}

Ao selecionar todas as equações, obtiveram-se as estimativas de altura de cada parcela amostral, empregando-as aos modelos genéricos aos tradicionais.

A análise de variância (Quadro 8) indicou que há interação significativa entre os fatores e entre as suas interações. Como neste estudo o interesse foi saber qual tratamento é estatisticamente diferente do outro, procedeu-se ao desdobramento apenas da interação entre os fatores parcelas $x$ tratamentos, conforme apresentado no Quadro 9.

Observando o Quadro 9, verifica-se que os tratamentos apresentaram comportamento diferenciado da testemunha, ou seja, pelo menos um dos seis tratamentos diferiu da testemunha. Dessa forma, aplicouse o teste de médias de Scott e Knott para verificar quais tratamentos diferiam da testemunha. Os resultados dos testes de média realizados em cada parcela são apresentados no Quadro 10.

Quadro 8 - Análise de variância do experimento com o povoamento de Eucalyptus grandis

Table 8 - Variance analysis for the experiment with Eucalyptus grandis stand

\begin{tabular}{lcccc}
\hline F. V. & G.L. & S.Q. & Q.M. & F \\
\hline Parcelas (P) & 21 & 8147,31 & 387,97 & $5,87 * *$ \\
Resíduo $_{\mathrm{A}}$ & 418 & 27632,59 & 66,11 & \\
Total $_{\mathrm{A}}$ & $(439)$ & $(35779,9)$ & $(454,08)$ & \\
Modelos (M) & 6 & 797,04 & 132,84 & $410,95^{*}$ \\
$\mathrm{P} x \mathrm{M}$ & 126 & 1634,06 & 12,97 & $40,12^{* *}$ \\
Resíduo $_{\mathrm{B}}$ & 2508 & 810,81 & 0,32 & \\
\hline Total $_{\mathrm{B}}$ & 3079 & 39021,70 & & \\
\hline
\end{tabular}

Analisando os resultados do Quadro 10, podese verificar que o ajuste de modelos hipsométricos tradicionais por parcela é o procedimento que apresenta melhor desempenho, visto que em $90,9 \%$ dos ajustes os resultados foram estatisticamente semelhantes ao da testemunha. Também é possível verificar que o modelo genérico teve ajuste com eficiência em $77,3 \%$ dos casos, sendo esse porcentual indicativo de que tal modelo pode ser aplicado no povoamento em substituição aos demais modelos.

Quadro 9 - Análise de variância do desdobramento da interação parcelas $x$ modelos

Table 9 - Variance analysis for the partitioning of the interaction plots $x$ treatments

\begin{tabular}{ccccc}
\hline F. V. & G.L. & S.Q. & Q.M. & F \\
\hline Parcela 1 & 6 & 47,63 & 7,94 & $24,56^{*}$ \\
Parcela 2 & 6 & 82,46 & 13,74 & $42,52^{*}$ \\
Parcela 3 & 6 & 90,45 & 15,08 & $46,64^{*}$ \\
Parcela 4 & 6 & 116,35 & 19,39 & $59,99^{*}$ \\
Parcela 5 & 6 & 42,89 & 7,15 & $22,11^{*}$ \\
Parcela 6 & 6 & 9,61 & 1,60 & $4,96^{*}$ \\
Parcela 7 & 6 & 136,11 & 22,69 & $70,18^{*}$ \\
Parcela 8 & 6 & 243,18 & 40,53 & $125,39^{*}$ \\
Parcela 9 & 6 & 140,71 & 23,45 & $72,55^{*}$ \\
Parcela 10 & 6 & 147,22 & 24,54 & $75,90^{*}$ \\
Parcela 11 & 6 & 413,70 & 68,95 & $213,30^{*}$ \\
Parcela 12 & 6 & 89,57 & 14,93 & $46,18^{*}$ \\
Parcela 13 & 6 & 151,88 & 25,31 & $78,31^{*}$ \\
Parcela 14 & 6 & 47,94 & 7,99 & $24,72^{*}$ \\
Parcela 15 & 6 & 119,56 & 19,93 & $61,65^{*}$ \\
Parcela 16 & 6 & 148,49 & 24,75 & $76,56^{*}$ \\
Parcela 17 & 6 & 52,29 & 8,71 & $26,96^{*}$ \\
Parcela 18 & 6 & 55,74 & 9,29 & $28,74^{*}$ \\
Parcela 19 & 6 & 87,16 & 14,53 & $44,94^{*}$ \\
Parcela 20 & 6 & 49,68 & 8,28 & $25,61^{*}$ \\
Parcela 21 & 6 & 61,16 & 10,19 & $31,53^{*}$ \\
Parcela 22 & 6 & 97,30 & 16,22 & $50,17^{*}$ \\
\hline ERRO & 2508 & 810,81 & 0,32 & \\
\hline & & & & \\
\hline
\end{tabular}

Quadro 10 - Teste de médias de Scott e Knott das estimativas médias das alturas Table 10 - Scott \& Knott's mean test for height mean estimates

\begin{tabular}{|c|c|c|c|c|c|c|c|}
\hline Parcela & Tratamento & Média* & Agrupamento & Parcela & Tratamento & Média* & Agrupamento \\
\hline \multirow[t]{7}{*}{1} & 6 & 19,027 & $\mathrm{a}$ & 2 & 6 & 19,722 & $\mathrm{a}$ \\
\hline & 5 & 19,201 & $\mathrm{a}$ & & 5 & 20,554 & $\mathrm{~b}$ \\
\hline & 3 & 19,683 & $\mathrm{~b}$ & & 3 & 20,593 & $\mathrm{~b}$ \\
\hline & 7 & 19,798 & $\mathrm{~b}$ & & 7 & 21,012 & $\mathrm{c}$ \\
\hline & 2 & 19,798 & $\mathrm{~b}$ & & 4 & 21,685 & d \\
\hline & 1 & 19,864 & $\mathrm{~b}$ & & 1 & 21,916 & d \\
\hline & 4 & 20,990 & c & & 2 & 21,916 & d \\
\hline
\end{tabular}

R. Árvore, Viçosa-MG, v.28, n.6, p.845-854, 2004 
Uso de diferentes alternativas para viabilizar a ...

Quadro 10 - cont.

Table 10 - cont

\begin{tabular}{|c|c|c|c|c|c|c|c|}
\hline Parcela & Tratamento & Média* & Agrupamento & Parcela & Tratamento & Média* & Agrupamento \\
\hline \multirow[t]{7}{*}{3} & 6 & 15,551 & $\mathrm{a}$ & 4 & 6 & 16,771 & $\mathrm{a}$ \\
\hline & 1 & 16,974 & $\mathrm{~b}$ & & 7 & 17,408 & $\mathrm{~b}$ \\
\hline & 7 & 16,977 & $\mathrm{~b}$ & & 2 & 17,408 & $\mathrm{~b}$ \\
\hline & 2 & 16,977 & $\mathrm{~b}$ & & 1 & 17,466 & $\mathrm{~b}$ \\
\hline & 5 & 17,722 & $\mathrm{c}$ & & 5 & 18,278 & $\mathrm{c}$ \\
\hline & 4 & 17,873 & $\mathrm{c}$ & & 4 & 19,050 & d \\
\hline & 3 & 18,165 & $\mathrm{~d}$ & & 3 & 19,465 & $\mathrm{~d}$ \\
\hline \multirow[t]{7}{*}{5} & 6 & 20,191 & $a$ & 6 & 6 & 20,494 & $\mathrm{a}$ \\
\hline & 4 & 21,243 & $\mathrm{~b}$ & & 4 & 20,767 & a \\
\hline & 7 & 21,243 & b & & 7 & 20,975 & b \\
\hline & 2 & 21,243 & $\mathrm{~b}$ & & 2 & 20,975 & $\mathrm{~b}$ \\
\hline & 1 & 21,243 & $\mathrm{~b}$ & & 5 & 20,975 & b \\
\hline & 3 & 21,535 & b & & 1 & 21,002 & $\mathrm{~b}$ \\
\hline & 5 & 22,222 & $\mathrm{c}$ & & 3 & 21,434 & $\mathrm{c}$ \\
\hline \multirow[t]{7}{*}{7} & 4 & 19,666 & $\bar{a}$ & 8 & 3 & 20,019 & $\mathrm{a}$ \\
\hline & 6 & 20,138 & a & & 6 & 20,367 & a \\
\hline & 3 & 20,234 & b & & 4 & 21,327 & b \\
\hline & 7 & 21,966 & $\mathrm{~b}$ & & 1 & 23,131 & $\mathrm{c}$ \\
\hline & 5 & 21,966 & $\mathrm{c}$ & & 2 & 23,132 & $\mathrm{c}$ \\
\hline & 2 & 21,966 & $\mathrm{c}$ & & 5 & 23,132 & $\mathrm{c}$ \\
\hline & 1 & 22,012 & $\mathrm{c}$ & & 7 & 23,132 & $\mathrm{c}$ \\
\hline \multirow[t]{7}{*}{9} & 4 & 20,817 & $\bar{a}$ & 10 & 3 & 19,048 & $\bar{a}$ \\
\hline & 3 & 21,376 & b & & 6 & 19,928 & b \\
\hline & 6 & 21,649 & $\mathrm{~b}$ & & 4 & 20,503 & $\mathrm{c}$ \\
\hline & 5 & 22,413 & $\mathrm{c}$ & & 1 & 21,740 & d \\
\hline & 1 & 23,389 & d & & 2 & 21,742 & d \\
\hline & 2 & 23,390 & d & & 5 & 21,742 & d \\
\hline & 7 & 23,390 & $\mathrm{~d}$ & & 7 & 21,742 & $\mathrm{~d}$ \\
\hline \multirow{7}{*}{11} & 6 & 17,805 & $a$ & 12 & 6 & 19,986 & $a$ \\
\hline & 4 & 18,201 & b & & 3 & 20,418 & b \\
\hline & 3 & 18,514 & b & & 5 & 20,891 & $\mathrm{c}$ \\
\hline & 1 & 20,362 & $\mathrm{c}$ & & 4 & 21,505 & d \\
\hline & 7 & 21,855 & d & & 2 & 22,060 & $\mathrm{e}$ \\
\hline & 5 & 21,855 & d & & 7 & 22,060 & $\mathrm{e}$ \\
\hline & 2 & 21,855 & d & & 1 & 22,081 & $\mathrm{e}$ \\
\hline \multirow[t]{7}{*}{13} & 6 & 19,877 & $\mathrm{a}$ & 14 & 6 & 18,929 & $\mathrm{a}$ \\
\hline & 1 & 20,431 & b & & 5 & 19,454 & b \\
\hline & 7 & 20,431 & $\mathrm{~b}$ & & 4 & 20,197 & $\mathrm{c}$ \\
\hline & 2 & 20,431 & b & & 1 & 20,399 & $\mathrm{c}$ \\
\hline & 3 & 20,431 & b & & 7 & 20,399 & $\mathrm{c}$ \\
\hline & 5 & 22,068 & $\mathrm{c}$ & & 2 & 20,399 & $\mathrm{c}$ \\
\hline & 4 & 22,979 & $\mathrm{~d}$ & & 3 & 20,681 & $\mathrm{c}$ \\
\hline \multirow[t]{7}{*}{15} & 6 & 16,612 & $\bar{a}$ & 16 & 6 & 18,140 & $\bar{a}$ \\
\hline & 7 & 17,483 & b & & 7 & 18,537 & b \\
\hline & 5 & 17,483 & b & & 5 & 18,537 & b \\
\hline & 2 & 17,483 & b & & 2 & 18,537 & $\mathrm{~b}$ \\
\hline & 1 & 17,506 & b & & 1 & 18,548 & b \\
\hline & 4 & 19,057 & $\mathrm{c}$ & & 4 & 20,469 & $\mathrm{c}$ \\
\hline & 3 & 19,421 & $\mathrm{~d}$ & & 3 & 20,937 & $\mathrm{~d}$ \\
\hline \multirow[t]{7}{*}{17} & 6 & 16,750 & $\mathrm{a}$ & 18 & 6 & 17,576 & $\mathrm{a}$ \\
\hline & 4 & 18,281 & b & & 1 & 18,563 & b \\
\hline & 1 & 18,457 & b & & 7 & 18,564 & $\mathrm{~b}$ \\
\hline & 7 & 18,459 & $\mathrm{~b}$ & & 2 & 18,564 & $\mathrm{~b}$ \\
\hline & 5 & 18,459 & $\mathrm{~b}$ & & 4 & 18,828 & b \\
\hline & 2 & 18,459 & $\mathrm{~b}$ & & 3 & 19,293 & c \\
\hline & 3 & 18,691 & $\mathrm{~b}$ & & 5 & 19,753 & $\mathrm{~d}$ \\
\hline
\end{tabular}

R. Árvore, Viçosa-MG, v.28, n.6, p.845-854, 2004 
Quadro 10-cont.

Table 10 - cont.

\begin{tabular}{|c|c|c|c|c|c|c|c|}
\hline Parcela & Tratamento & Média* & Agrupamento & Parcela & Tratamento & Média* & Agrupamento \\
\hline \multirow[t]{7}{*}{19} & 6 & 18,346 & $\mathrm{a}$ & \multirow[t]{7}{*}{20} & 6 & 15,614 & $\mathrm{a}$ \\
\hline & 7 & 19,385 & b & & 7 & 17,020 & $\mathrm{~b}$ \\
\hline & 5 & 19,385 & b & & 5 & 17,020 & $\mathrm{~b}$ \\
\hline & 2 & 19,385 & b & & 2 & 17,020 & $\mathrm{~b}$ \\
\hline & 3 & 19,676 & b & & 1 & 17,033 & $\mathrm{~b}$ \\
\hline & 1 & 20,675 & c & & 4 & 17,384 & $\mathrm{c}$ \\
\hline & 4 & 20,833 & $\mathrm{c}$ & & 3 & 17,651 & $\mathrm{c}$ \\
\hline \multirow[t]{7}{*}{21} & 6 & 16,405 & $\bar{a}$ & \multirow[t]{7}{*}{22} & 6 & 15,650 & $\bar{a}$ \\
\hline & 2 & 17,647 & $\mathrm{~b}$ & & 1 & 17,445 & $\mathrm{~b}$ \\
\hline & 1 & 17,663 & $\mathrm{~b}$ & & 7 & 17,446 & $\mathrm{~b}$ \\
\hline & 5 & 18,068 & $\mathrm{c}$ & & 2 & 17,446 & $b$ \\
\hline & 4 & 18,179 & c & & 5 & 17,997 & $\mathrm{c}$ \\
\hline & 7 & 18,444 & d & & 4 & 18,052 & $\mathrm{c}$ \\
\hline & 3 & 18,471 & d & & 3 & 18,440 & $\mathrm{~d}$ \\
\hline
\end{tabular}

*Médias seguidas por letras iguais maiúsculas na coluna não diferem entre si $(\mathrm{p}<0,05)$.

Em que: Tratamento $1=$ testemunha (altura real), Tratamento $2=$ modelo hipsométrico tradicional por parcela, Tratamento $3=$ modelo hipsométrico tradicional por idade, Tratamento $4=$ modelo hipsométrico tradicional por sítio, Tratamento $5=$ modelo hipsométrico tradicional para o agrupamento de sítio e idade, Tratamento $6=$ modelo hipsométrico tradicional para o agrupamento das parcelas e Tratamento $7=$ modelo hipsométrico genérico.

\section{CONCLUSÕES}

Após as análises dos resultados, tornaram-se possíveis as seguintes conclusões:

- O ajuste por parcela utilizando modelos tradicionais é o procedimento ideal para estimar a altura das árvores.

- Os bons resultados obtidos com o ajuste de modelos genéricos indicaram o potencial de sua utilização na estimativa das alturas das árvores.

\section{REFERÊNCIAS BIBLIOGRÁFICAS}

\author{
ABREU, E. C. R. Modelagem da prognose \\ precoce do volume por classe \\ diamétrica para Eucalyptus grandis. 2000. \\ 70 f. Dissertação (Mestrado em Engenharia \\ Florestal) - Universidade Federal de Lavras, \\ Lavras, 2000.
}

BARROS, D. A. et al. Comportamento de modelos hipsométricos tradicionais e genéricos para plantações de Pinus oocarpa em diferentes tratamentos. Boletim de Pesquisa Florestal, v. 45, p. 3 - 28, 2004.

R. Árvore, Viçosa-MG, v.28, n.6, p.845-854, 2004
CARDoso, D. J. Avaliação da influência dos fatores sítio, idade, densidade e posição sociológica na relação hipsométrica para Pinus taeda nas regiões central e sudoeste do estado do Paraná. 1989. 106f. Dissertação (Mestrado em Engenharia Florestal) - Universidade Federal do Paraná, Curitiba, 1989.

CARDOSO, D. J. et al. Avaliação da influência dos fatores idade e sítio na relação hipsométrica para Pinus taeda nas regiões central e Sudoeste do estado do Paraná. Revista Floresta, v. 19, n. 1-2, p. 96 - 115, 1989.

FERREIRA, D. F. SISVAR: sistema de análise de variância para dados balanceados, versão 4.0. Lavras: DEX/UFLA, 1999. (Software estatístico)

SAS INSTITUTE. The SAS system, version 6.11 Cary: 1996. (Software estatístico)

SCOLFORO, J.R.S. Mensuração florestal 3: relações quantitativas em volume, peso e a relação hipsométrica. Lavras: ESAL/FAEPE, 1993. 292 p.

SCOTT, A. J.; KNOTT, M. A Cluster analysis method for grouping means in the analysis of variance. Biometrics, v. 30, p. 505-512, 1974. 